\title{
CAPSULE COMMENTARIES \\ Capsule Commentary on Wright et. al.: Reduced Effectiveness of Interruptive Drug-Drug Interaction Alerts After Conversion to a Commercial Electronic Health Record
}

\author{
Ellen E. Sarcone, MD \\ Denver Health Medical Center, University of Colorado School of Medicine, Aurora, CO, USA.
}

J Gen Intern Med 33(11): 1954

DOI: $10.1007 / \mathrm{s} 11606-018-4510-y$

(c) Society of General Internal Medicine 2018

$\mathrm{W}$ right and colleagues examined the quantity of drugdrug interaction (DDI) alerts received by physicians in a legacy electronic health record (EHR) that incorporated a homegrown DDI database with those alerts received after implementation of Epic integrated with a commercial drug knowledge database (KB). They found that DDI alerts increased sixfold after Epic implementation. Acceptance rate of the alerts, defined as discontinuing one of the interacting medications, fell substantially even for DDIs considered to be the most risky or clinically significant. The increased frequency of alerts and the different manner in which clinicians were alerted in the EHR (hard stop versus soft stop and interruptive versus passive) probably both contributed to the decline in acceptance rate in this study.

Institutions with EHRs have adopted clinical decision support (CDS) tools such as DDI alerts for patient safety reasons and to meet Meaningful Use (an EHR incentive program) requirements. ${ }^{2}$ Many institutions use commercial KBs that integrate with the EHR; there is a high level of variability in the number of DDI alerts generated from the three most widely used KBs in the USA. ${ }^{3}$ Because of the high frequency of DDI alerts, they are overridden up to $90 \%$ of the time by physicians and advanced practice providers. Alert fatigue and lack of integration of specific patient information in the CDS systems are thought to contribute to the frequency with which alerts are bypassed.
Clinical decision support within the EHR was developed to prompt best practices and promote patient safety. DDI alerts are only one of many different alerts in the EHR. There is flexibility in how to set up DDI alerts but a paucity of standard recommendations on the optimal deployment of DDI alerts to avoid alert fatigue while preserving patient safety. There is also poor crossover in the knowledge bases most commonly used in the USA. ${ }^{3}$ We should work diligently with our EHR and KB vendors to limit DDI alerts to only those which are clinically significant for the patient and ensure the deployment of alerts in the EHR clearly indicates the potential severity of the interaction.

Corresponding Author: Ellen E. Sarcone, MD; Denver Health Medical Center University of Colorado School of Medicine, Aurora, CO, USA (e-mail: ellen.sarcone@dhha.org).

\section{Compliance with ethical standards:}

Conflict of interest: The authors declare that they do not have a conflict of interest.

\section{REFERENCES}

1. Wright A, Aaron S, Seger DL, Samal L, Schiff GD, Bates DW. Reduced Effectiveness of Interruptive Drug-Drug Interaction Alerts after Conversion to a Commercial Electronic Health Record. J Gen Intern Med. DOI: https://doi.org/10.1007/s11606-018-4415-9.

2. https://www.cms.gov/Regulations-and-Guidance/Legislation/ EHRIncentivePrograms/index.html?redirect=/EHRIncentivePrograms

3. Fung KW, Kapusnik-Uner J, Cunningham J, Higby-Baker $\mathbf{S}$, Bodenreider 0. Comparison of Three commercial Knowledge Bases for Detection of Drug-Drug Interactions in Clinical Decision Support. J Am Med Inform Assoc. 2017;34(4):806-12

4. Humphrey K, Jorina M, Harper M, Dodson B, Kim SY, Ozonoff A. An Investigation of Drug-Drug Interaction Alert Overrides at a Pediatric Hospital. Hospital Pediatrics 2018; 8(5)293-99: 